\title{
NEW DIRECTIONS IN RESEARCH ON DOMINANT DESIGNS
}

\author{
JOHANN PETER MURMANN \\ Kellogg School of Management, Northwestern University, Evanston, Il 60201, USA \\ KOEN FRENKEN \\ URU, Utrecht University, The Netherlands
}

\begin{abstract}
Our theory provides an unambiguous definition of dominant designs (stable core components that can be stable interfaces) and the inclusion of multiple levels of analysis (system, subsystems, components). We introduce the concept of an operational principle and offer a systematic definition of core and peripheral subsystems based on the concept of pleiotropy.

\section{INTRODUCTION}

Since Abernathy and Utterback (1978) first developed the concept of a dominant design from a study of the automobile industry, many writers in the field of organization theory and strategy have found the concept an extremely useful tool for studying the evolution of technological products. At the heart of dominant design thinking lies the empirical observation that technology evolves by trial and error and thus entails risks for the population of firms engaged in its development. The only way to reduce the uncertainty about technological potential and user needs is to create different designs and wait for feedback from users. Over time, only one or a few designs from the much larger number of design trials will succeed. The firms that happen to be producers of the winning designs will flourish, whereas firms that invested in the failing designs will incur economic losses and may even go out of business. The dynamics that lead to dominant designs are of central importance to firms that have a stake in the way technology evolves because the emergence of a dominant design produces winners and losers. A review of all the empirical studies concerned with dominant designs (Murmann and Frenken 2002) revealed that research is far from codified and standardized and that scholars disagree on definitions of dominant design, units of analysis and boundary conditions of their theories. We argue that part of the apparent inconsistencies surrounding dominant design research is caused by a lack of analytical concepts. We propose a complex systems approach in an attempt to reconcile, at least to an extent, the major inconsistencies in current research on dominant design.
\end{abstract}

\section{TECHNOLOGY AS A COMPLEX SYSTEM}

Following the work of Simon (1962) on the evolution of complex systems, and in line with work by Rosenberg (1969), we define a technology as a man-made system that is constructed from components that function collectively to produce a number of functions for users. Simon (1962) pointed out that artifacts, like other complex systems, are not just made up of elementary components, all directly interacting with one another, but rather consist of a nested hierarchy of subsystems. An entire airplane, for example, is made up of a fuselage, wings, propelling device, and landing gear, which can be represented as first-order subsystems. Each of these first-order subsystems has potentially included within it smaller second-order subsystems, and potentially many further levels of ever-smaller subsystems until the level of the fundamental 
or basic components is reached. In the case of an airplane, its wing subsystems contain within flaps, fuel tanks, lights, etc. The flaps, in turn, are composed of steering flaps and breaking flaps. Flaps have a lattice and a skin and are held together by flush rivets. Each level in the artifact hierarchy can go through its own technology cycle marked by the processes of variation, selection, and retention (Tushman and Murmann 1998).

To come to any meaningful definition of a dominant design, one needs to define the class of designs in which a design can be said to be dominant. Without delineation, any conceptualization of a dominant design is impossible because dominance can only be established with reference to a distribution of artifacts that belong to a technological class. We believe the concept of an operational principle is a very useful tool for classifying artifacts into classes. This concept, originally developed by Polanyi (1962) in the context of developing a theory of how human beings know things, was later used in Vincenti's (1990) writings on the history of airplane. For Polanyi, an operational principle captures the kind of knowledge a human designer must have in order to build a technological device that works on the physical world in a desired way. To put it differently, an operational principle defines how the parts interact with one another to implement the goal of overall technology.

Consider the example of the principle underlying the first successful human flight. Instead of trying to design a flying machine with flapping wings to provide both the counterforce to gravity and forward thrust, Cawley in 1809 (Vincenti, 1990) proposed to separate lift from propulsion by using a fixed wing and propelling it forward with motor power. The central idea was that moving a rigid surface through resisting air would provide the upward force countering gravity. As Vincenti has noted, this was a radically different way to conceptualize the design of an airplane because it freed designers from the impractical idea of flapping wings. Subsequently, the fixed-wing and forward propulsion idea became the operational principle underlying all airplane designs.

When human beings have grasped the operational principle of a technology, they know how an artifact can act on nature in a special beneficial way. Because an operational principle essentially specifies how components need to be arranged in order to create a successful artifact, operational principles reveal the abstract logic of how an artifact works and thus provide the starting point for understanding what the essential aspects of a particular technology are. In fact, the operational principle of an artifact sets out the relevant dimensions (also called the design space of an artifact). For the researchers of technological change this means that once the operational principle of an artifact has been determined, this automatically decides the key technical dimensions of an artifact and thus determines in what dimensions two artifacts can differ technically without belonging to different classes of technology.

In using the concept of an operational principle, one is able to compare different technologies by probing whether they work according to the same the operational principle or not. For instance, planes and helicopters, both devices for air travel, differ in terms of how they achieve the general task of transporting humans in the air. A plane accomplishes flight by separating the propelling function and the lifting function into two separate components (the propeller or jet and the wings), whereas the helicopter realizes movement in the air by implementing the lifting and propelling function in one and the same component, the large vertical rotor. Rockets, another class of devices for traveling, make air travel possible by allowing an expanding air-fuel mix to escape only through the rear of the device and thus propel it forward. Rocket propulsion requires neither wings nor propellers. 
Synopsis I: To organize the universe of all technological artifacts into general product categories, the operational principle of the artifact is a useful classification criterion.

The example of the three technology classes enabling air travel also serves to demonstrate a second feature of complex artifacts that we discussed earlier: hierarchical structure. Devices to travel through air are classified as aircraft, helicopters, rockets, and others. Within the class of aircraft, one can distinguish between propeller aircraft and jet aircraft. In propeller aircraft, one can distinguish between piston propeller and turbopropeller, and so forth. Complex systems typically consist of components that are themselves complex systems. This hierarchical view of dominant designs implies that there can, in fact, be dominant designs at a higher (more encompassing) level without there being any dominant design at the lower level. All that is required is that the operational principles-i.e., the way lower-level subsystems are combined into a system through a set of standard design rules-be dominant across the industry (see Baldwin and Clark, 2000, for a superb articulation of the idea of design rules).

Synopsis II: To classify artifacts within a product class, one can use the operational principles of the artifact's subsystems, starting with the first-order subsystems all the way to the lowest level subsystems.

To state that a dominant design is present in, say, aircraft, when all aircraft share the same design for all their components, would count as a very stringent requirement that never would be met in practice. The history of technological innovation shows that, over time, design converges in some dimensions yet differentiates in others. One solution to this definitional problem is to argue that dominant designs can only be assessed at the level of a single component (subsystem). However, given the hierarchical nature of complex technological artifacts, the shift of analysis from the artifact to its first-level subsystem also shifts the problem of defining a dominant design to that next level.

We have come to the conclusion that the answer to this question lies in the notion of distinguishing the core and peripheral components of any design. To define core and periphery in complex artifacts, the concept of pleiotropy from biology provides analytic sharpness. In biology, researchers distinguish between the genotype in which mutation occurs (genes) and the phenotype at the level in which differential success of organisms becomes apparent (traits). The number of traits affected by a particular gene in the genotype is referred to as a gene's "pleiotropy" (Altenberg, 1995). The concept of pleiotropy in biology has been of great importance to the understanding of evolution: a mutation in a gene of high pleiotropy has a much lower probability of success than a mutation in a gene with low pleiotropy (Frenken 2001 and forthcoming). The probability that a mutation will increase fitness depends inversely on the pleiotropy of the gene in which the mutation occurs. A mutation in a gene with high pleiotropy means that the fitness values of many traits are assigned new values, the joint net effect of which is unlikely to be advantageous, whereas a mutation in a gene with low pleiotropy implies that only few traits are assigned new fitness values.

The most important insight, which holds also true for the structure of biological evolution, is that once a design has settled on particular variants of core components, further advances tend to be concentrated in peripheral components only (Altenberg 1995; Frenken, forthcoming). It follows that even in the case of modular products, a dominant design can exist, as defined by the high-pleiotropy elements: the interface standards. As long as particular 
interface standards dominate an industry, one can speak of a dominant design, even if all modules exist in hundred kinds of varieties. The structuring nature of high-pleiotropy elements as an insight is certainly not new. Clark (1985) already noted this pattern of problem solving in the automobile history. Early efforts in automobile design were concentrated mainly on the operational principles of different car technologies, and on their respective core subsystems and components with high pleiotropy. These included the kind of engine to use (steam, electric, gasoline), the kind of steering device (wheel, tillers), and the kind of material to use (metal, wood). In particular, the central problem at the time concerned the reliability of cars with different fuels, an experimental stage that can be considered a period of competing core components. Once design converged to a fixed set of core concepts components (gasoline engines, steering wheels, and metal bodies), the design of core components were no longer subject to dispute, and innovations shifted towards low-pleiotropy peripheral components to finetune very specific functions (lamps, belt, seats, interior, catalyst, and so on) and to incrementally refine the core technologies underlying the core components (pistons, fuel inlet, and so on). As Clark (1985, 243) put it: "the technical agenda was set for a variety of subsidiary problems and choices. (...) But such things would have had no place on the agenda established by the electric car. There the relevant focus for supporting technology would have included the chemistry of batteries and the parameters of electric motors.”

It is important to stress that although changes in core components have very low probability of success, this does not imply that these changes will never occur. What it does mean is that a change in a core component that initially leads to poorer performance needs to be accompanied by many additional changes in other components, possibly even by architectural innovations in the way components are organized into a whole. This explains why changes in core components of a technology take so much time and effort before they are successfully introduced. It also explains why, at any level of an artifact hierarchy, technology cycles are triggered by the substitution of core components.

Synopsis III: A dominant design is defined by the choice of high-pleiotropy components. Synopsis IV: A core component is more difficult to change successfully than a peripheral component.

\section{A REFINED MODEL DOMINANT DESIGNS}

On the basis of our conceptualization of technology as a complex hierarchical system, we are now in the position to articulate a model of dominant designs as a nested hierarchy of technology cycles. A dominant design exists in a technological class when the majority of designs have the same technologies for the high-pleiotropy core components. Interfaces can constitute such core components because interfaces can be high-pleiotropy elements in a complex system. Pleiotropy thus determines which subsystems/interfaces are core and which are peripheral. The notion of a majority of designs can be defined empirically by using either a threshold (e.g., 50 percent) measure or a variety measure (e.g., entropy; Herfindahl index). The geographic areas and industries covered in the empirical analysis need to be identified. The problems noted in the introduction concerning the unit of analysis, the granularity of the analysis and the boundary conditions can also be solved. Because artifacts can be conceptualized as complex hierarchical systems, there are multiple units of analysis. The overall artifact constitutes the system and its lower levels comprise a nested hierarchy of subsystems. To specify a unit of 
analysis unambiguously, one needs to define both the level above and the level below the focal unit of analysis. Because the range of levels between the two extremes of granularity can be very wide, the level of granularity adopted in a particular study needs to be specified by identifying what would count as one granularity level finer and what would be one granularity level coarser. Technologies differ in their speed of development. The boundary conditions, finally, follow from the complex systems perspective: Our model applies to complex artifacts. But just as Simon (1962) thought, we believe virtually all artifacts are complex systems because designers need to consider-even in the case of artifacts that look simple and composite-numerous relevant design dimensions that interact in complex and unpredictable ways.

\section{LINKING TECHNOLOGICAL TO INDUSTRIAL DYNAMICS}

Management scholars and industrial organization economists have been keenly interested in the dynamics of change in technological artifacts because the structure of artifacts has a profound effect on the organization of the industrial system that produces them. One can interpret the entire history of dominant design research as an attempt to find a theory that would map technological changes to changes in the industrial organization of firms and markets. Scholars have uncovered large amounts of evidence showing that technological changes powerfully shape the range of different organizational arrangement possible at any given moment. At the same time, though, market structures provide incentives for developing certain types of innovation over other types. Before a dominant design emerges, many small firms engage in product innovation in an attempt to develop and appropriate the knowledge underlying the future dominant design, or parts of it. After a dominant design emerges, and the industry has become concentrated, firms have an incentive to engage in process innovations, the costs of which can be spread over many products. This means that changes in technology and market structure are causally connected. Although many scholars recognize the importance of technical change in explaining industrial dynamics, many studies attempted to explain industrial dynamics solely from a demographic perspective (Carroll and Hannan, 2000; Klepper, 2002). This research strategy is probably motivated, at least in part, by the lack of a consistent nomenclature of technical change. One of the roadblocks lying in the way of making more progress in spelling out the causal links between technological innovation and industrial changes is precisely that empirical research on dominant designs has not consistently conceptualized technologies in terms of a hierarchical system and has not specified the unit of analysis of the particular research results reported. By using the hierarchical model formulated above, we believe researchers will be able to gather more systematic data to trace the organizational and strategic consequences of innovations at the different levels of the artifact structure.

\section{REFERENCES}

Abernathy, W.J., Utterback, J., 1978. Patterns of industrial innovation. Technology Review 50: 4147.

Altenberg, L., 1995. Genome growth and the evolution of the genotype-phenotype map, in: W. Banzhaf and F.H. Eckman (Editors), Evolution and Biocomputation (Springer-Verlag, Berlin and Heidelberg).

Baldwin, C.Y., Clark, K.B., 2000. Design Rules. Volume 1: The Power of Modularity (MIT Press, Cambridge, MA). 
Carroll, G.R., Hannan, M.T., 2000, The Demography of Corporations and Industries (Princeton University Press, Princeton, NJ).

Clark, K.B., 1985. The interaction of design hierarchies and market concepts in technological evolution. Research Policy 14: 235-251.

Frenken, K., 2001. Fitness landscapes, heuristics and technological paradigms: a critique on random search models in evolutionary economics. American Institute of Physics Conference Proceedings 573(1): 558-565.

Frenken, K., forthcoming, Innovation, Evolution and Complexity Theory (Cheltenham and Brookfield: Edward Elgar).

Klepper, S., 2002. The capabilities of new firms and the evolution of the US automobile industry. Industrial and Corporate Change 11(4): 645-666.

Murmann, J.P., Frenken, K., 2002, Towards a systematic framework for research on dominant designs, technological innovations, and industrial change. Papers on Economics and Evolution, 2002-12, Max Planck Institute, Jena.

Polanyi, M., 1962. Personal Knowledge: Towards a Post-Critical Philosophy (Harper \& Row, New York).

Rosenberg, N., 1969. The direction of technological change: inducement mechanisms and focusing devices. Economic Development and Cultural Change 18: 1-24.

Simon, H.A., 1962. The architecture of complexity: hierarchic systems. Proceedings of the American Philosophical Society 106 (December): 467-482.

Tushman, M.L, Murmann, J.P., 1998. Dominant designs, technology cycles and organizational outcomes, Research in Organizational Behavior 20: 231-266.

Vincenti, W.G., 1990. What Do Engineers Know and How Do They Know It? (Johns Hopkins Press, Baltimore). 
Copyright of Academy of Management Proceedings is the property of Academy of Management. The copyright in an individual article may be maintained by the author in certain cases. Content may not be copied or emailed to multiple sites or posted to a listserv without the copyright holder's express written permission. However, users may print, download, or email articles for individual use. 